\title{
LESIONS OF THE CILIARY GANGLION AS A CAUSE OF ARGYLL ROBERTSON AND ADIE PUPILS*
}

\author{
BY \\ MALCOLM E. CAMERON \\ Brisbane
}

MANY and varied are the lesions postulated for the Argyll Robertson pupil (Duke-Elder, 1949). Cord lesions, and lesions in the afferent pathway; midbrain, and efferent pathway, including the ciliary ganglion and iris, have all been suggested at various times. The difficulty of detecting small changes in nerves and brain at autopsy has probably accounted for this.

Adie's pupil, on the other hand, has not excited so much comment, probably because no autopsy material has been forthcoming. The theories about the site of the lesion in cases of Adie pupil range from that of Lowenstein and Friedman (1942), suggesting the diencephalon, to that of Adler and Scheie (1940), suggesting the ciliary ganglion.

A case of Adie pupil following herpes zoster ophthalmicus is described below, and an attempt will be made to show that both the Adie and Argyll Robertson pupils have their origins in a ciliary ganglion lesion.

\section{Case Report}

A married woman aged 26 years, was first seen on May 6, 1956, with a very severe right herpes zoster ophthalmicus of 3 days' duration. There was marked vesiculation over the path of the right supra- and infratrochlear and supra-orbital nerves. The lids were grossly swollen and overriding each other and there was marked chemosis of the conjunctiva, with staining by fluorescein of a small horizontal patch in the centre of the cornea. The pupil was three-quarters dilated, because of previous treatment with atropine drops, the dilation being greater on the temporal side. The right fundus was normal. There was no impairment then or subsequently of the ocular motility, but a loss of corneal sensation was noted. Treatment was begun with atropine drops, cortisone drops, and chloramphenicol ointment.

On June 18, 1956 (6 weeks after the onset and while atropine was still being used), the right eye was white, with clear cornea and normal fundus. The right visual acuity (under atropine) was $6 / 5$ with correction $-1.5 \mathrm{D}$ sph., $+0.5 \mathrm{D}$ cyl., axis $60^{\circ}$. The atropine was stopped.

6 weeks after stopping the atropine and 3 months after the onset, the right pupil was still irregularly dilated and did not react to light directly or consensually, or to accommodation.

On March 12, 1957, 10 months after the onset of the herpes zoster, the patient reported that the right pupil, which had previously been immobile, was now changing its size. She had noticed this for the first time only a few days before when she looked into her mirror.

Examination.-The right pupil was irregularly dilated, with its long axis from 10 to 5 o'clock. It measured about $6 \times 5 \mathrm{~mm}$. and did not react to light directly

\footnotetext{
* Received for publication September 17, 1958.
} 
or consensually (Fig. 1).

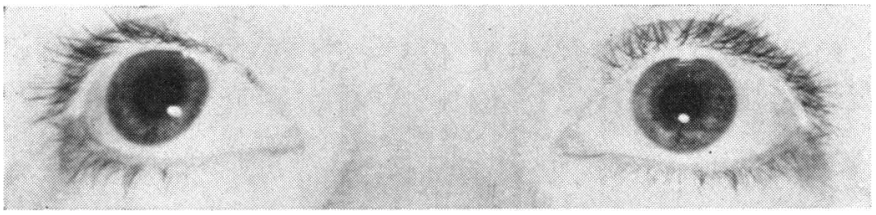

FIG. 1.-No mecholyl; bright light; looking at infinity; showing irregular dilation of right pupil and normal contraction of left pupil to light. The scarred area of the right forehead is not very obvious, because, on account of the dark brown irides, the development time of the prints had to be lengthened.

However, it reacted quite noticeably to convergence but not to any other movement of the eyes (Fig. 2). The normal left pupil was $3 \mathrm{~mm}$. in diameter in average light and reacted normally to light directly and consensually and to accommodation.

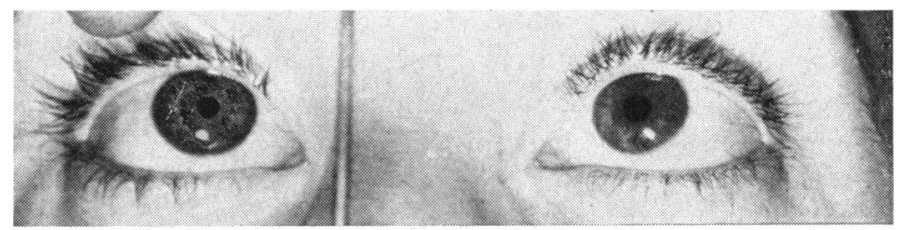

Fig. 2.-No mecholyl; dim light; looking at near; right pupil smaller than left pupil and slightly irregular.

The uncorrected visual acuity was $6 / 24$ and $\mathrm{J} 1$ (most) in the right eye and 6/12 and $\mathrm{J} 1$ in the left. With correction $-1 \mathrm{D}$ sph., $-0.5 \mathrm{D}$ cyl., axis $180^{\circ}$ to each eye, the visual acuity was $6 / 5$ (most) and $J 2$ in the right eye and 6/4 and J1 in the left.

It was thought that there might have been a very slight paralysis of accommodation, as nothing else could be seen to account for the lowered right near vision, which could be restored to normal by adding $+1 \mathrm{D}$ sph. A few months later, the corrected visual acuity for both near and distance was equal and normal; 6/4 and J1, right and left. The right and left central and peripheral fields to $2 / 1000$ and $2 / 330$ white objects were normal.

There was much scarring over the affected area (Fig. 1) and there had been much constitutional upset during the past months, with severe headache, sleeplessness, and depression. The knee jerks were present and active and the blood Wassermann reaction was negative (000).

Pharmacological Reactions.-The reactions of the pupils to various drugs (three applications of one drop made at 3-minute intervals) were as follows:

(1) 10 PER CENT. NeO-SYNEPHRINE.-The right pupil, 35 minutes after starting, had dilated uniformly and completely to $8 \mathrm{~mm}$. The left pupil dilated only to $5 \mathrm{~mm}$. and reacted to light directly and consensually and to accommodation. The right pupil did not react to light directly or consensually when maximally dilated, but did react to near, contracting to about $6 \mathrm{~mm}$.

(2) 5 PER CENT. COCAINE.-Both pupils dilated equally and uniformly to $8 \mathrm{~mm}$. in 30 minutes. The right eye was more dilated than its fellow 12 hours later and resumed its usual size in another 24 hours. The left resumed its normal size in 2 hours.

(3) 1 PER CENT. Homatropine.-The right pupil only was tested. It dilated to $7 \mathrm{~mm}$. in 30 minutes, but in doing so, changed its long axis from 10 to 5 to 1 to 7 o'clock. The temporal edge remained perfectly circular, while the nasal edge was irregular. It returned to its usual size in about 60 hours. 
(4) 2.5 PER CENT. MeChOLYL. -30 minutes after three instillations, the right pupil was smaller than the left (Fig. 3).

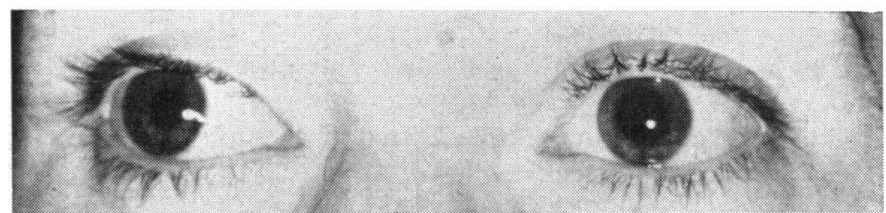

Fig. 3.-After mecholyl; dim light; looking at infinity; mecholyl contracts right pupil but has no effect on normal left pupil.

The irregularity of the right pupil was more pronounced, with a circular temporal edge and a flattened nasal edge. The right pupil reacted to near after the mecholyl but not to light directly or consensually. The right pupil at the end of the near reaction was much smaller than the left and its irregularity was accentuated, with its long axis from 10 to 4 o'clock (Fig. 4). The left pupil was unaffected in size or in reactions by the mecholyl.

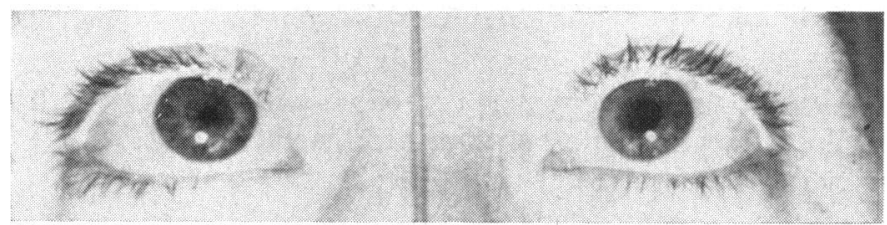

FIG. 4.-After mecholyl; dim light; looking at near; right pupil smaller than left pupil.

(5) 1 PER CENT. PILOCARPINE.-30 minutes after the first instillation, the right pupil was smaller than the left $(1.5$ and $2 \mathrm{~mm}$. respectively). It was flattened above and still reacted to near slightly but not to light. 6 to 8 hours later, the right pupil had regained its usual size.

(6) 0.25 PER CENT. ESERINE.-The right pupil only was tested. 30 minutes after the first instillation, the right pupil measured $1.5 \times 1.0 \mathrm{~mm}$. in diameter and was flattened above. It did not react to light at any time during its contraction. It was still miotic 24 hours later, but resumed its usual size 60 hours after testing. The near reaction was maintained during contraction by eserine, at least until the pupil measured $3 \times 2 \mathrm{~mm}$. The patient volunteered that during the eserine miosis the post-herpetic neuralgia was much improved, but that it recurred with its former intensity when the miosis passed. It is difficult, however, to see that this could be anything but coincidental.

Darkness. -45 minutes in darkness did not appreciably dilate the affected pupil. It was not possible, unfortunately, to photograph it by electronic flash and so compare it with the normal size, but at least one can say that there was no marked difference. Nor was the light reflex restored by bringing the patient into bright daylight.

Synkinesis.-There was no pupillary movement on moving the eyes in any direction, except on convergence.

Slit-lamp Examination and the Light Reflex.-There was no obvious atrophy of the iris. Apart from the irregularity of the margin of the pupil nasally and its greater size in bright light compared with the left, it looked normal. There was, however, a loss of the pigment frill nasally from 2 to 5 o'clock. The pigment frill was complete in the normal left eye and its absence may be indicative of sphincter atrophy in this area.

On flicking the lamp on and off, while the slit was aimed at different parts of the pupillary border, it was seen that there was a very slight pupillary contraction of the large arc from 2 to 5 o'clock, maximum movement being at 7 o'clock. This reaction could not be observed by the unaided eye, so that if magnification were not used, the light reflex would appear to be absent. 
Near Reflex.-Since the pupil did not react to light, a pupillogram of the convergence reaction in artificial light was attempted, using 16- $\mathrm{mm}$. ciné film and 32 frames/sec. The latency period for contraction and dilation could not be measured, but it was found that while contraction was brisk and occupied about $2.5 \mathrm{sec}$., the dilation was greatly prolonged and took about $11 \mathrm{sec}$. These relative differences could also be appreciated clinically.

At the end of the contraction phase, the affected right pupil was smaller than the left and its irregularity was more noticeable.

\section{Discussion}

From the shape and size differences with various drugs, the following deductions may be made:

(1) Most of the light fibres have been destroyed. The very small reaction at 7 o'clock to the very bright direct slit-lamp light may be due to stimulation of a few surviving light fibres, which still retain some connexions in the ciliary ganglion.

(2) Because its shape is oval with the long axis from 10 to 5 o'clock, and because the arc 10 to 7 to 5 o'clock is circular, compared with the irregularity of the arc 10 to 2 to 5 o'clock, it can be inferred that there has been considerable destruction of the sphincter muscle nasally but very little, if any, temporally.

(3) The greater size of the pupil is partly due to the nasal sphincter lesion and partly to undue irritability of the sympathetic, as shown by extreme dilation with cocaine and neo-synephrine.

(4) The reaction to homatropine, in which the temporal sector dilated circularly while the nasal sector was hardly affected, also points to selective destruction of the sphincter. It will be remembered that the irregularity of the right pupil was noticed at the height of the infection (during atropine therapy), so that the sphincter destruction occurs very early.

(5) The reaction of the pupil to convergence for the first time 10 months after the onset of the herpes, indicates that regeneration of the near fibres has taken place in this time. It resembles the case of François, as described by Naquin (1954), in which the pupil first reacted to near 6 months after the onset of herpes zoster. There has not been any regeneration of the light fibres in either of these cases and this lends support to the belief that the light fibres, but not the near fibres, relay in the ciliary ganglion. It would appear that the near fibres, which pass through but do not relay in the ciliary ganglion, were destroyed initially in the attack but subsequently regenerated. The fibres to the ciliary muscle were not involved to any extent and neither were those fibres supplying any of the extraocular muscles, including the inferior oblique, the nerve of which lies very close to the ciliary ganglion. This may be because of the larger and more numerous nerve fibres supplying these muscles. According to Tatlow (1952) the incidence of palsies of one or more extra-ocular muscles in herpes zoster ophthalmicus is about 7 per cent.

(6) The prompt, complete, and circular dilation to neo-synephrine and cocaine show that the sympathetic nerve fibres and the dilator muscle are intact, although the exaggerated reaction of the affected pupil to 10 per cent. neo-synephrine points to a sensitization to nor-adrenalin. 
In considering such a case as the above, which showed severe involvement of the naso-ciliary nerve and short ciliary nerves, it would seem impossible that the dilator fibres would escape severe damage. However, they have largely escaped, and the reason may lie in the double innervation from the sympathetic system, viz. through the sympathetic root of the ciliary ganglion, from the carotid plexus on the one hand and on the other hand from the long ciliary branches of the nasociliary, derived also from the carotid sympathetic plexus in the cavernous sinus.

(7) The sensitization of the pupil to mecholyl indicates post-ganglionic destruction of the parasympathetic pathways. The reaction to pilocarpine was also greater in the right (affected) pupil than in the left and, with both mecholyl and pilocarpine, the characteristic deformity of the pupil (flattening of the long 10 to 2 to 5 o'clock arc and preservation of the circular shape of the shorter 10 to 7 to 5 o'clock arc) was seen.

This post-herpetic pupil thus bears a striking resemblance to an Adie pupil (the Strasburger type) in the following respects:

(1) It has an absent light reflex.

(2) It has a prolonged near reaction, especially the second part.

(3) It is larger than its fellow.

(4) It exhibits sensitivity to mecholyl (and to neo-synephrine).

Most observers have placed the disturbance in Adie's syndrome in the central sympathetic system, but a peripheral origin has been suggested by Adler and Scheie (1940) as a result of their discovery of the sensitivity of the Adie pupil to mecholyl. The above case shows an Adie pupil produced by undoubted peripheral changes and offers strong evidence for a peripheral, as against a central, origin in this condition.

Naquin (1954) has described a pupil occurring after herpes zoster ophthalmicus which was similar in all respects to the classical Argyll Robertson pupil. It was small, reacted briskly to convergence, and dilated rapidly. It did not react to light and dilated incompletely with atropine. Tests for syphilis were negative.

No doubt if sufficient cases of post-herpetic pupillary abnormalities were studied, a complete gradation from the typical Argyll Robertson pupil to the many different types of Adie pupil could be found. Unfortunately, Tatlow (1952) did not study in detail the six atypical Argyll Robertson pupils which he reported as occurring after herpes zoster ophthalmicus. There is no need to postulate two separate efferent pathways for pupillary constriction (Nathan and Aldren Turner, 1942). It is enough to postulate that the light fibres are more vulnerable than the near fibres, because they synapse in the ciliary ganglion whereas the near fibres do not synapse. The synkinetic movements of the pupil after section of the third nerve (Bender, 1945) can be explained by the fibres of the extra-ocular muscles achieving synapse with the cells of the final relay of efferent light fibres, through the branch the inferior oblique contributes to the ciliary ganglion. 
An infection such as herpes zoster can thus affect differentially the light fibres, the near fibres, and the dilator fibres. If the light fibre synapses are destroyed together with the majority of dilator fibres, an Argyll Robertson pupil is produced. If the convergence fibres are only slightly damaged, with almost complete light fibre synapse damage in the ciliary ganglion, and minimal dilator fibre damage, the various types of Adie pupil are produced.

\section{Summary}

(1) A case of Adie pupil following herpes zoster ophthalmicus is described.

(2) The pharmacological reactions are detailed and a sensitivity to mecholyl and neo-synephrine is shown.

(3) It is thought that this case offers strong evidence that an Adie pupil can be produced by a lesion in the ciliary ganglion.

(4) A similar origin for the Argyll Robertson pupil is considered likely, both the Adie and Argyll Robertson pupils being considered as variants of a destructive lesion in the ciliary ganglion.

My grateful thanks are due to Dr. B. Klug who kindly referred the patient; to Dr. B. Wilson for the pupillary photographs; to the University of Queensland Photographic Department; to the Brisbane Clinic Research Foundation for financial help; and finally to the patient herself, who suffered calmly many inconveniences.

\section{REFERENCES}

Adler, F. H., and Scheie, H. G. (1940). Trans. Amer. ophthal. Soc., 38, 183.

BENDER, M. B. (1945). Arch. Neurol. Psychiat. (Chicago), 53, 418.

DUKE-ELDER, S. (1949). "Text-book of Ophthalmology", vol. 4, p. 3782. Kimpton, London.

Lowenstein, O., and Friedman, E. D. (1942). Arch. Ophthal. (Chicago), 28, 1042.

NAQuin, H. A. (1954). Amer. J. Ophthal., 38, 23.

Nathan, P. W., and Aldren Turner, J. W. (1942). Brain, 65, 343.

TATLOW, W. F. T. (1952). J. Neurol. Neurosurg. Psychiat., 15, 45. 